\title{
Correction to: Climate Change and Agriculture in India: Impact and Adaptation
}

\author{
Syed Sheraz Mahdi
}

\section{Correction to:}

S. Sheraz Mahdi (ed.), Climate Change and Agriculture

in India: Impact and Adaptation, https://doi.org/10.1007/978-3-319-90086-5

The author group of the chapters 18 and 19 were inadvertently revised in the original publication. The author groups of these chapters have now been restored as in the original submission. 